\title{
Relaciones intergeneracionales en la construcción social de la percepción del riesgo*
}

\author{
DAYRA ELIZABETH OJEDA ROSERO \\ Y ESPERANZA LÓPEZ VÁZQUEZ
}

En las ciencias sociales, en general, y en la psicología social, en particular, existe especial interés por comprender cómo los grupos humanos asumen los riesgos de desastres. En este artículo se analizan las interacciones entre familia, comunidad y sociedad en el proceso de construcción social de la percepción del riesgo de desastre a partir de las relaciones intergeneracionales. El propósito es aportar fundamentos teórico-conceptuales y estratégicos al tema. Se sugiere la necesidad de aprovechar las relaciones intergeneracionales para la gestión de los riesgos y de colocarlas en un ámbito político y en la sociedad en general.

PALABRAS CLAVE: construcción social del riesgo, desastre, percepción del riesgo, relaciones intergeneracionales, gestión de riesgos de desastres

\section{Intergenerational Relationships in the Social Construction of Risk Perception}

In the social sciences in general and in social psychology in particular, there is special interest in understanding how human groups are positioned against disaster risks. This article describes the interactions between family, community and society in the process of social construction of the perceived risk of disasters, from intergenerational relationships perspective. The purpose of this work is to provide theoretical, conceptual and strategic bases to risk problems. It is suggested to take advantage of intergenerational relationships for managing risk, besides positioning them at the political field and in general society.

DAYra ElizABEth OJeda Rosero

Universidad de Nariño, Pasto,

Nariño, Colombia

deliza75@yahoo.com

ESPERANZA LÓPEZ VÁZQUEZ

Universidad Autónoma del Estado de Morelos,

Cuernavaca, Morelos, México

esperanzal@uaem.mx
KEYWORDS: social construction of risk, disaster, risk perception, intergenerational relations, disaster risk management

Trabajo derivado de la tesis de doctorado en psicología, Construcción intergeneracional de la percepción del riesgo volcánico, Centro de Investigación Transdisciplinar en Psicología, Universidad Autónoma del Estado de Morelos, con apoyo del Consejo Nacional de Ciencia y Tecnología de México. 


\section{Introducción}

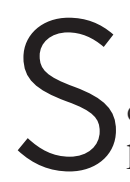

e ha demostrado que el concepto construcción social es útil para el análisis de

los riesgos de desastres (García, 2005). Hacking (1999; 2001) afirma que al hablar de construcción social se busca generar conciencia de una realidad considerada natural y familiar, pero que no siempre fue de esa manera, pues ha sufrido transformaciones en el tiempo. Desde esta perspectiva, los riesgos de desastres, entendidos como construcciones sociales, resultan de procesos históricos, políticos e ideológicos, entre otros.

El presente ensayo habla de la construcción social en el ámbito de los riesgos de desastres y resalta la interacción de varias generaciones como uno de los factores implicados en su configuración. Este aspecto se ha considerado de manera implícita, pero se ha analizado poco en los estudios relacionados con el riesgo y los desastres. La atención se centra en las dimensiones subjetivas, y en particular, en las percepciones que se forman en contextos de interacción social con una amenaza, como la erupción de un volcán, inundaciones, sismos y tsunamis, entre otras. Por lo anterior, se parte de la teoría acerca de la construcción social de la realidad (Berger y Luckman, 1968) y del enfoque sociocultural de los riesgos (Douglas y Wildavsky, 1982; Douglas, 1996; Beck, 2010) para entender mejor la subjetividad de los actores sociales (Ferrari, 2010).

\section{Riesgo de desastre y construcción social del riesgo}

El riesgo es un constructo complejo que para los fines de este trabajo puede entenderse como la posibilidad de daños o pérdidas económicas, sociales o ambientales, dada la interacción entre amenaza — peligro- y vulnerabilidad — exposición al peligro y potencial de afectación-. Por su parte, el desastre, concepto también complejo, puede verse como un proceso social, delimitado en un espacio y un 
tiempo, resultado de un fenómeno de origen natural, socionatural o antrópico. En un desastre, con base en las condiciones de vulnerabilidad, se presentan alteraciones importantes en la población — en la vida, la salud, las pertenencias, el ambiente- $-\mathrm{y}$ es necesaria una respuesta inmediata de las autoridades y de la población misma, así como la movilización de recursos externos para afrontarlo (Maskrey, 1997; Cardona, 2003; Lavell, 2005).

Entender el desastre como proceso implica analizar las condiciones sociales y naturales en las que emerge, es decir, tener un profundo conocimiento de la población y su historia en el territorio, aspectos que aluden a la "construcción social del riesgo" y a la noción del "ciclo o continuo del riesgo" del cual el desastre es un momento (Lavell, 2005: 30). El riesgo de desastre es un fenómeno que involucra factores ambientales y geológicos, pero también económicos, políticos, culturales, sociales y psicológicos (Slovic, 2000; Briones, 2005; Wachinger y Renn, 2010; Wachinger et al., 2013). Por ende, el concepto de construcción social del riesgo de desastre requiere diálogos entre diversas disciplinas y saberes populares.

En Latinoamérica, a finales del siglo Xx y comienzos del actual, creció el interés por el aporte de las ciencias sociales al tema de los riesgos de desastres (Lavell, 1993; 2005). Sin embargo, este interés requiere aún afianzarse en el ámbito institucional para ser coherente con los frecuentes desastres a los que la humanidad se expone (Cardona, 2007; Cardona et al., 2010; IPCC, 2014), por ello es necesario seguir insistiendo en su estudio y aplicación desde la academia.

En las últimas décadas, el discurso sobre el riesgo como construcción social relacionado con el desarrollo y su gestión para reducir desastres ha penetrado en instancias internacionales, nacionales y locales (Gellert de Pinto, 2012). En Latinoamérica existen ejemplos de políticas coherentes con esta concepción, como la Estrategia Andina para la
Prevención y Atención de Desastres (Consejo Andino de Ministros de Relaciones Exteriores, 2009) y la Política Centroamericana de Gestión Integrada de Riesgo de Desastres (Cepredenac, 2010). También en diversos trabajos académicos se comprende el riesgo como una construcción social (Douglas, 1996; García, 2005; Lavell, 2005; López, 2011; Beck, 2010; Urteaga y Eizagirre, 2013; Castillo, 2014; Tierney, 2014).

El concepto construcción social del riesgo contiene tres variables básicas: las amenazas físicas que provocan los desastres, los procesos sociales como causas de la vulnerabilidad y los mecanismos cognitivos por medio de los cuales se define lo que es riesgoso y aceptable en una sociedad (Beck, 2010; Douglas, 1996; Tierney, 2014).

De acuerdo con García (2005), los enfoques del concepto de construcción social del riesgo aplicado a los desastres pueden asociarse a la percepción y la vulnerabilidad. En Latinoamérica, a partir de la década de 1980, en las ciencias sociales se ha conformado una tendencia científica y social que plantea la construcción social la mayoría de las veces como construcción material o vulnerabilidad. También se contempla la construcción discursiva y simbóli$\mathrm{ca}$, en la cual destacan los estudios culturalistas que analizan la influencia de la cultura en la concepción y postura frente a los riesgos aceptables, y los amplios estudios sobre percepciones y representaciones (Douglas, 1996; Castro y Zusman, 2009).

\section{Relaciones intergeneracionales}

Como preámbulo al tema de las relaciones intergeneracionales, es importante hablar de las generaciones, las cuales han sido objeto de estudio a lo largo de la historia en áreas del saber, como la sociología, la literatura, la filosofía, entre las principales. Existen investigaciones acerca de sus representantes más destacados y su desarrollo (e.g. Laín, 1945; Donati, 
1999; Martin, 2008; Leccardi y Feixa, 2011; Testaverde, 2012, Caballero y Baigorri, 2013; Lüscher et al., 2015).

El concepto de generación, según Donati (1999), trae consigo dos líneas interpretativas desarrolladas en los estudios modernos y contemporáneos. La primera, surgida entre los siglos XIX y XX, es la generación como grupo de edad. Se plantea un sentido histórico que alude a grupos que comparten la misma experiencia significativa dada su cercanía de edad. La segunda línea interpretativa, que se puede situar en la década de 1980, hace referencia a la generación como descendencia familiar-parental. Donati (1999) identifica diferencias entre generación, como cohorte — sentido demográfico-, grupo de edad —sentido histórico-, unidad generacional - subgrupo involucrado en movimientos sociales-y generación en sentido sociológico - personas que comparten relaciones de descendencia socialmente mediadas-.

Leccardi y Feixa (2011: 13) mencionan tres momentos históricos en el desarrollo del concepto de generación: a) entre las guerras mundiales, cuando se fundaron las bases filosóficas sobre la sucesión y la coexistencia generacional; b) en la década de 1960, cuando el trabajo se centró en el conflicto generacional, y c) desde la década de 1990, dado el surgimiento de la sociedad en red, con la teoría novedosa sobre lapso generacional, en relación con la experticia de los jóvenes en la tecnología digital en comparación con las generaciones anteriores.

Según Lüscher et al. (2015), hay tres fases en la historia del concepto. La primera, en la Antigüedad y la Edad Media, se fundamenta en la necesidad de comprender el presente a partir del pasado y la tradición, reconoce la transmisión de conocimiento de generación en generación. La segunda corresponde a la Edad Moderna, etapa en la que el concepto de generación adquiere un sentido distinto, puesto que señala el comienzo de un futuro nuevo y del progreso. La tercera etapa, más reciente, equipara una generación a un fragmento de tiempo, el futuro es algo incierto, pues hay pérdida de certezas, y se presenta una influencia mutua entre la familia y la sociedad. Es decir, nos encontramos en una época de incertidumbre y de complejas relaciones internas y externas a la familia.

Para Donati (1999) las generaciones deben comprenderse en sentido relacional, pues involucran tanto la descendencia como la ascendencia familiar y la mediación de las relaciones sociales externas a la familia. Desde esta perspectiva, deben combinarse los roles con la edad social, por ejemplo, hijos jóvenes o hijos adultos, abuelos jóvenes o abuelos mayores. Hay que tener en cuenta que una generación define su identidad con base en la identidad familiar mediada por factores sociales como la economía, la política y la cultura.

El término generación se asocia a un grupo de personas que comparten características que lo identifican y a su vez lo diferencian de otras generaciones. Este proceso depende de diversos aspectos, entre ellos las influencias del momento histórico y los acontecimientos vividos, el ámbito geográficoterritorial, los valores y la época cultural-educacional experimentada. Estos contextos condicionan el perfil, el carácter y la personalidad de una generación mediante un proceso de sinergia que permite que ésta contribuya con su comunidad, lo cual se conoce como impronta generacional (Gutiérrez y Herráiz, 2009: 25).

La aceptación social de los riesgos, como correlato de la percepción social, define cómo una sociedad se interpreta a sí misma en un momento histórico determinado (Douglas y Wildavsky, 1982; Douglas, 1996) y depende de los consensos y disensos en los significados que las generaciones otorguen a los peligros que enfrentan. Si cada generación tiene una impronta - sello o marca- (Gutiérrez y Herráiz, 2009), se espera encontrar patrones comunes ante el riesgo, mas no se puede homogeneizar a todos los individuos y grupos sociales, pues en las 


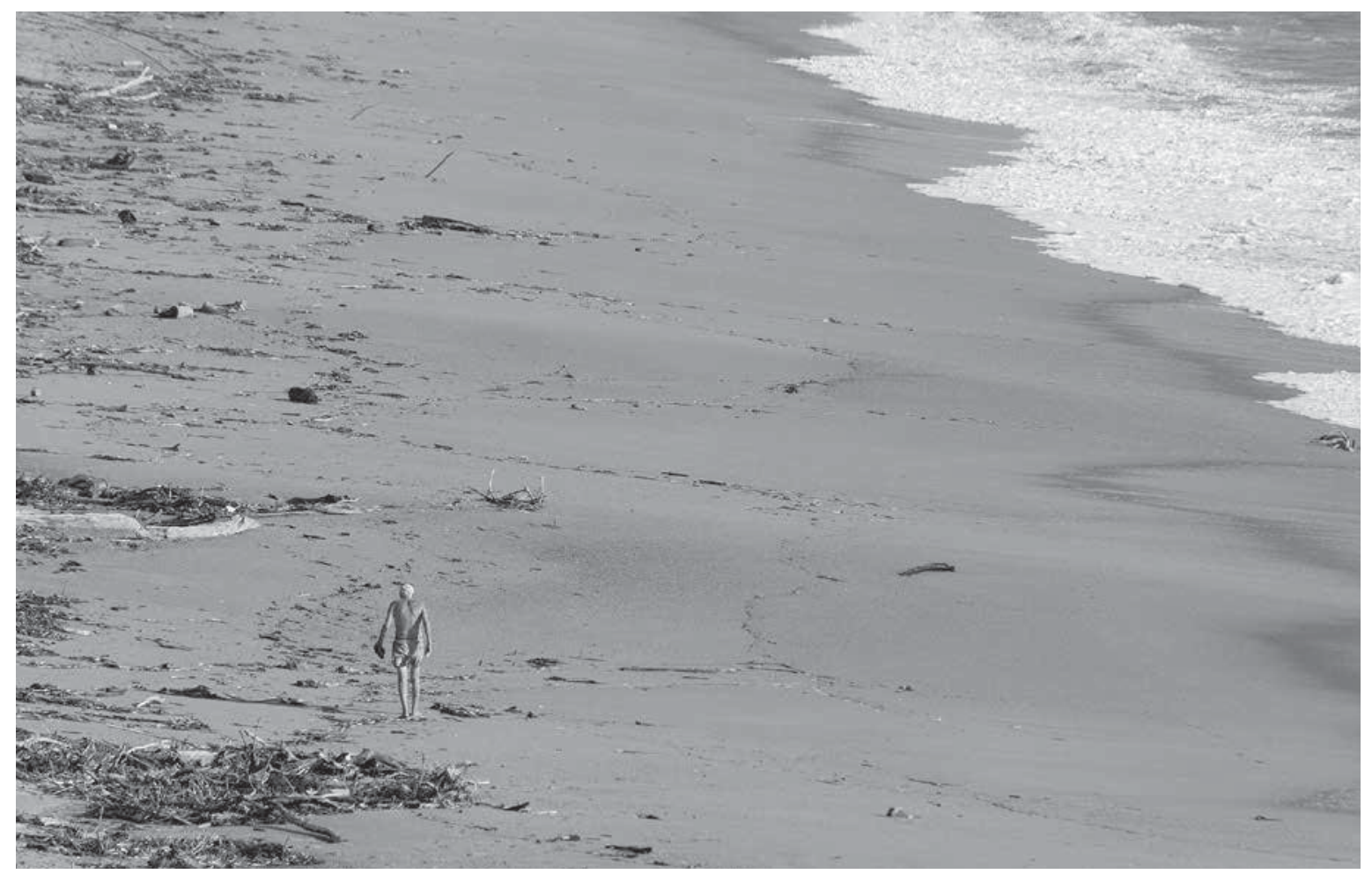

Prometeo Lucero Un turista camina solo en la playa de Barra de Navidad, Jalisco, después del paso del huracán Patricia, 25 de octubre de 2015.

interacciones entre diferentes generaciones, tanto pasadas como presentes, se crean patrones colectivos e individuales frente al riesgo, así como puntos de desencuentro.

\section{Relaciones intergeneracionales y familia}

Después de identificar elementos fundamentales de la noción de generación, es necesario analizar las relaciones intergeneracionales. Para Falcke y Wagner (2003), lo intergeneracional significa influencia recíproca, pero en detrimento de la permanencia de ciertos procesos familiares en las generaciones sucesivas. En el presente trabajo, nos interesan las relaciones intergeneracionales entendidas como la influencia mutua de varios actores sociales. Por lo tanto, a diferencia de la perspectiva de Falcke y
Wagner (2003), se concibe la posibilidad tanto de continuidad como de cambio de los referentes a lo largo de la historia familiar y social.

Sáez (2009) manifiesta que la expresión intergeneracional ha adquirido más fuerza que la noción de generación y es más utilizada en ámbitos como el social y el político, sin que por esto se pueda afirmar que sea un término claro o unívoco. No obstante, lo intergeneracional representa mejor una postura relacional antes que sustancialista o esencialista acerca de las generaciones. En este sentido, no sólo remite a las edades de la vida sino a los encuentros y la supervivencia de un "nosotros".

Para Sánchez (2009), en las últimas décadas ha habido un interés creciente por las relaciones entre las generaciones, dada la segregación por edades presente en muchas sociedades. En la década de 1990, la intergeneracionalidad se plantea como un elemento 
clave para el desarrollo comunitario por medio de la cohesión y la sostenibilidad de las comunidades, en el ánimo de promover una ciudadanía activa. Quizá no es que no exista la intergeneracionalidad, sino que es importante visibilizarla y analizar sus implicaciones.

En el fondo, lo fundamental es el convencimiento de que "todo ser humano es intergeneracional” (Sánchez, 2009: 10), pues lleva consigo el cruce de tres tiempos: presente, pasado y futuro. En este sentido de transcurso del tiempo, se hace referencia a procesos intergeneracionales para acentuar su connotación histórica y la conexión con los contextos social, político, económico y cultural, entre otros que enmarcan las relaciones intergeneracionales.

La familia es uno de los principales ámbitos socializadores, con la presencia de roles particulares - por ejemplo, abuelos, padres y nietos- que contribuyen a entender la formación y desarrollo de las relaciones intergeneracionales. Donati (1999) señala que sociológicamente no se puede hablar de generación sin hacer referencia a la familia, pues los ciclos de vida individual, familiar y generacional, además de estar interrelacionados, se condicionan mutuamente. A partir de las relaciones intergeneracionales puede producirse tanto continuidad como cambios en los patrones de comportamiento, que se denominan trayectorias o transiciones (Caballero, 2014).

En una misma familia, las generaciones interactúan entre sí. Al respecto, Schutz (1974) propuso cuatro categorías: el predecesor, el contemporáneo, el asociado y el sucesor. El predecesor ya no vive y sólo puede ser conocido por medio de referencias de otros, puede influir en los demás, pero no viceversa; persiste en el discurso de quienes lo conocieron y lo nombran, por lo tanto, el conocimiento de este antepasado es indirecto. Entre ellos hay personas como los bisabuelos o abuelos difuntos que se convierten en transmisores del tiempo y el espacio pasados. El sucesor vivirá después de que el individuo muera, no comparte su tiempo ni su espacio. Las relaciones sociales, en su mayoría, se comparten con el contemporáneo con quien se tiene en común tiempo y espacio, y se mantienen relaciones cara a cara. Por último, el asociado es el contemporáneo con quien se comparte un tiempo y una relación cara a cara, con quien el tiempo avanza a la par; por ejemplo, la triada abuelos, padres y nietos. A partir de las relaciones entre estos actores generacionales hay procesos de continuidad de los marcos culturales de los ancestros o suceden transformaciones. A esto nos referimos a continuación.

\section{Reproducción de esquemas y cambio de patrones en la dinámica familia-sociedad}

Falcke y Wagner (2003) advierten que en el proceso de perpetuación de la identidad familiar de generación en generación intervienen varios factores: a) lealtades, relacionadas con el sentido ético del deber y la justicia, con el cual el individuo se siente comprometido como parte de una familia; b) valores, entendidos como marcos relevantes para las familias; c) creencias, como conjunto de presupuestos asociados a lo que es o no correcto y debe ser obedecido; d) mitos, como elementos sustanciales para la construcción de la realidad en la búsqueda de generar explicaciones para otorgar sentido a las ambigüedades y a partir de allí regular las relaciones dentro y fuera de la familia; e) secretos, que son actos o sentimientos ocultos o privados, contrarios a los patrones familiares; f) ritos o rituales, entendidos como ceremonias y actos simbólicos que representan la identidad familiar y permiten la transmisión de mitos y la educación de sus miembros, y g) legados, como proceso que hereda a las generaciones siguientes las principales características de la familia actual. Estos factores en conjunto posibilitan la perpetuación del patrimonio psíquico de la familia por medio de la educación (Wagner, Carmona y Falcke, 2003). 
Si bien lo anterior aporta a la comprensión de los procesos de reproducción social mediante el papel de la familia, necesita complementarse con la identificación de los mecanismos de cambio social. La interacción de la familia con la sociedad conlleva estas consecuencias y es necesario visualizarlas de manera integral para facilitar el aporte en la comprensión de los procesos de construcción social del riesgo. Dada la diversidad de las familias, así como su inevitable interacción con el entorno, es pertinente descifrar las interacciones que se forjan en el tejido familia-sociedad.

La transmisión de patrones hoy es discontinua. Si bien permanece, es más compleja, por las condiciones del mundo actual. Puede plantearse la coexistencia de formas de relaciones intergeneracionales en el mundo contemporáneo. En primer lugar, las formas tradicionales en las cuales los mayores enseñan a los más jóvenes las pautas de comportamiento socialmente aceptado que deben seguir de manera estricta para asegurar la reproducción de la cultura. En este modelo, el cambio social resulta difícil o al menos lento. En otras formas, se introduce el aprendizaje entre pares en cualquier edad de la vida, lo que moviliza el cambio social. Por último, aparece una forma importante de relación en la que los más pequeños ejercen influencia en el comportamiento de los mayores, circunstancia visible en la actualidad cuando los nativos de la era digital enseñan esta tecnología a los mayores, además de otros comportamientos valorados en la sociedad de hoy.

Lo anterior corresponde a lo que Margaret Mead (1970) llamó las culturas posfigurativas, cofigurativas y prefigurativas, respectivamente. En el caso de Carles Feixa (2003), se ejemplifican con la "metáfora del reloj". Esto supone nuevos retos para cada generación, en su necesidad de reelaborar sus roles y sus formas de relación, que requieren posturas reflexivas para enfrentar el presente, el cual ya no está marcado por vínculos generacionales asimétricos (Nadorowsky, 2014).
Donati (1999) ya había manifestado que el enfoque de las relaciones intergeneracionales se fundamenta en los cambios en las interacciones entre la familia y la sociedad a lo largo de la historia, desde la rígida transmisión de patrones sociales de generación en generación hasta los tipos de relaciones entre familia y sociedad, en los que la esfera pública tiene una influencia cada vez mayor.

La familia es el entorno social, cultural e histórico en el que ocurre la socialización primaria, pero también es un espacio de confluencia de lo público y lo privado. Al observar las generaciones en una misma familia, pueden identificarse dinámicas sociales en distintos tiempos, al reconstruir el pasado y el presente mediante el significado que le dan quienes lo vivieron y lo viven. Esta dimensión temporal puede establecer puentes entre los procesos macroestructurales y los microestructurales, pues se entiende que la acción de individuos concretos produce relaciones sociales en un tiempo y un espacio (Donati, 1999). En este marco, se entrelazan tres tiempos: el tiempo generacional — categorías culturales del momento en un contexto histórico-, el tiempo individual — ciclo vital - y el tiempo familiar — expectativas que se arrastran de generaciones anteriores- (Caballero, 2014).

La televisión, la escuela y los grupos de pares tienen cada vez más incidencia en el individuo. Frente a ello, la familia se torna en cierto sentido impotente y parece que en cada ciclo vital las personas deben arreglárselas solas para avanzar, mientras no exista un sistema cultural que conecte de mejor manera a la familia con la sociedad (Donati, 1999). Además de la familia, hay otros referentes y modelos: la comunidad a la que se pertenece, la escuela, los medios de comunicación, los pares y otras personas significativas, quienes también son agentes de transmisión intergeneracional (Wagner, Carmona y Falcke, 2003). Estos agentes aparecen en escena en la construcción social del riesgo, de manera que será necesario tenerlos en cuenta en sus interacciones para la gestión de riesgos. 


\section{Enfoques en el estudio de las relaciones intergeneracionales}

Los estudios intergeneracionales posibilitan conocer las interacciones de la familia y la sociedad en las dimensiones macro y microsocial. Varios enfoques tienen en común el interés por las relaciones entre individuos y grupos en una generación o entre generaciones, pero se diferencian en el énfasis conceptual.

Lüscher etal. (2015) mencionan cinco grupos de estudio acerca de las relaciones intergeneracionales: a) las generaciones genealógicas, es decir, los parentescos, los ancestros y los roles sociales; b) las generaciones pedagógicas, en referencia a las relaciones y los roles educativos; c) las generaciones socioculturales e históricas, relacionadas con guerras y otros conflictos sociales, movimientos culturales, seguridad social y Estado de bienestar; d) las generaciones como etiquetas, para caracterizar grupos específicos de población. Esta clasificación resume las principales áreas de desarrollo de los estudios generacionales.

Se encontró también que algunos estudios se refieren a la transmisión intergeneracional en tópicos como la violencia; por ejemplo, el maltrato infantil, la pobreza o las desigualdades sociales (Ramírez, 2003; Nina, Grillo y Alonso, 2003; Moreno, 2011). La transmisión intergeneracional se comprende como "la repetición de patrones de comportamiento, en las condiciones de haber experimentado, o haber observado cualquiera de estas acciones en miembros de familia de origen o de comunidad" (Ramírez, 2003: 57).

Otra línea de trabajo acerca de la transmisión intergeneracional se refiere a los comportamientos positivos, como las habilidades sociales (Braz et al., 2013), los usos y costumbres, u otras experiencias de aprendizaje (Méndez y Castro, 2011; Botero, Vega y Orozco, 2012; Ortelli, 2012). También podrían clasificarse los estudios sobre la transmisión entre generaciones, como los realizados desde el enfoque psicodinámico, como el trabajo de Werba (2002) acerca de los secretos y los duelos ancestrales, y el de Nussbaum (2009) sobre las identificaciones alienantes y la repetición.

Encontramos también estudios que se aproximan al acercamiento cultural intergeneracional, que puede entenderse como "el vínculo que se da a través de algún componente cultural (valores, prácticas, acciones, etc.) entre personas pertenecientes a distintas generaciones" (Gallego, 2011: 18). En esta línea, se pueden hallar trabajos sobre las relaciones entre abuelos y nietos (González y De la Fuente, 2008; Cruz y Acosta, 2009; Sarasty-Almeida, González-Gómez y Velasco-Charfuelán, 2014). Un ejemplo es Gallego (2011), que estudia las interrelaciones entre mayores y más jóvenes, y encuentra que los estereotipos y los medios de comunicación influyen en los desencuentros entre estas generaciones a raíz de que pueden propiciar un desconocimiento del otro.

Por otra parte, están los estudios focalizados en la comunicación intergeneracional. Por ejemplo, el trabajo de Harwood, quien realizó un análisis de la comunicación intergeneracional entre extraños y entre miembros de la familia y reveló que las identidades marcadas por la edad son cruciales en los tipos de comunicación intergeneracional, pues la "experiencia de comunicación intergeneracional es fundamentalmente distinta si se analiza dentro o fuera del contexto de la familia" (2002: 80).

En Costa Rica se realizó una investigación para validar un cuestionario y medir la percepción de la comunicación intergeneracional. Se encontraron diferencias significativas en la percepción de la comunicación según el grupo etario. Por los resultados obtenidos, los autores sugieren la necesidad de “investigar más detenidamente las particularidades culturales de la comunicación intergeneracional en Latinoamérica desde la perspectiva de la comparación cultural, tomando en cuenta las dimensiones 
clásicas del individualismo y el colectivismo" (Pérez-Sánchez y Smith-Castro, 2008: 600).

Un estudio hecho en Colombia mostró las relaciones interinstitucionales e intergeneracionales entre maestros y alumnos en una escuela pública urbana, de las cuales se derivan las figuras de maestro-alumno y adulto-joven, respectivamente (López-Moreno y Alvarado, 2011). En México, Caballero (2014) realizó una investigación sobre relaciones intrageneracionales e intergeneracionales con mujeres, con base en historias de vida. Estudió los cambios y continuidades en sus prácticas y discursos, en las trayectorias y transiciones de vida de tres generaciones de mexicanas - abuelas, madres y nietas-, en torno a educación y profesión, matrimonio o unión, y maternidad.

El interés por los procesos intergeneracionales es evidente, así como la diversidad en su concepción y tratamiento desde varias disciplinas de las ciencias sociales. Esto es motivo para desarrollar nuevas investigaciones en las cuales se retomen las generaciones y las relaciones intergeneracionales como temas fundamentales para entender los procesos implicados en la dinámica de continuidad y cambio familiar y social en Latinoamérica y el mundo. En este contexto, se requiere hacer estudios sobre riesgos de desastre para mejorar la comprensión de los aspectos psicosociales y culturales involucrados en su construcción social.

\section{Construcción social de la percepción del riesgo}

La percepción es un factor relevante en el estudio de los riesgos de emergencias y desastres. Diversos autores coinciden en que la percepción influye en el proceso de construcción de los riesgos. En este sentido, Tierney (2014) plantea que las creencias, actitudes y valores compartidos construyen realidades y proveen parte del orden social, al mismo tiempo que mantienen las actividades humanas consistentes y de alguna manera predictibles. Si consideramos que las personas constantemente interpretamos los estímulos ambientales, estudiar las percepciones del riesgo es importante porque éstas influyen en las decisiones que la gente toma (Slovic, 2000; Fischhoff y Kadvany, 2013).

La percepción de riesgo se entiende como un proceso psicológico que comprende una operación compleja, en la cual intervienen la selección, organización e interpretación de información (Slovic, 2000). La percepción del riesgo se comprende como el conjunto de aspectos individuales y colectivos - cognitivos, afectivos, socioculturales, políticos, entre otros- que interactúan en el proceso de interpretación de un peligro potencial y la asignación de significados al entorno (Douglas, 1996; Fischhoff et al., 2000; Siegrist y Cvetcovitch, 2000; Sjöberg, 2000; Slovic y Weber, 2002; Beck, 2002; 2008; Vera, 2009; Fischhoff y Kadvany, 2013; Urteaga y Eizagirre, 2013).

Cuando el énfasis se pone en los factores sociales y culturales que intervienen en este proceso, se habla de percepción social. Desde esta perspectiva, la percepción del riesgo está determinada por factores como el grupo al que se pertenece, la cultura y los marcos de interpretación de la realidad (Vargas, 1994). En ese sentido, la percepción no es un proceso objetivo e individual sino más bien subjetivo y social. Para estudiar la percepción del riesgo en las ciencias sociales, se requiere analizar actitudes, creencias, juicios y sentimientos, así como valores y disposiciones sociales y culturales frente a una amenaza o peligro, y los beneficios que éstas representan (Sabogal et al., 2006).

Entonces, para comprender la percepción del riesgo hay que superar posturas unidimensionales y apostarle a otras que relacionen disciplinas, como la sociología, la antropología, la psicología social o las ciencias políticas, entre otras, según las cuales el individuo no es la única ni la principal unidad de análisis. Este enfoque es conocido como sociocultural 


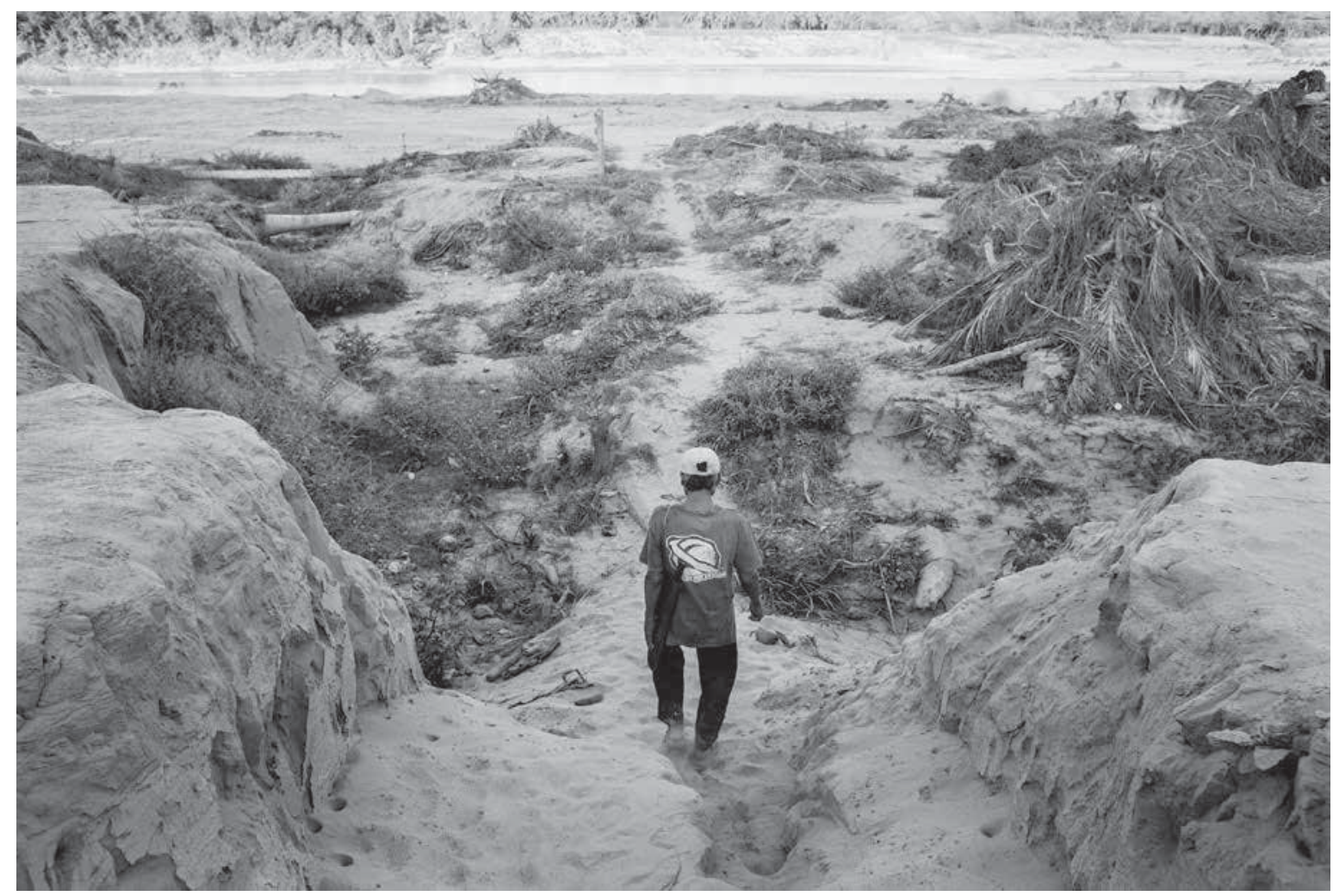

Prometeo Lucero - Un campesino camina en los bordes del río Papagayo después del huracán Manuel. Aguacaliente, Acapulco, Guerrero, 2013.

y se ha utilizado como base para demostrar las diferencias entre distintas culturas frente a la percepción del riesgo. No existe una interpretación estándar de la realidad social, cada grupo social posee sus propias concepciones. Douglas (1996) afirma que los desastres dependen en gran medida de las interpretaciones mediadas por la atención selectiva, que se basa en la estructura social como sistema moral. Para esta autora, una sociedad acepta o no determinados riesgos en cada momento histórico. Douglas y Wildavsky (1982) analizaron los riesgos tecnológicos en la sociedad moderna, estudiaron cómo nuestras sociedades aceptan diversos peligros, se hacen más tolerantes a ellos y a la vez reflejan los aspectos socialmente aceptados en un momento histórico.

$\mathrm{Al}$ considerar la percepción del riesgo como un proceso social, cada población elige sus riesgos, minimiza algunos y maximiza otros. En esta selección rara vez intervienen argumentos científicos. Las características culturales, las cosmovisiones y los patrones de interacción social influyen en la percepción selectiva de los riesgos (Douglas, 1996; Beck, 1998; Douglas y Wildavsky, 1982).

Urteaga y Eizagirre (2013: 167) plantean la relación entre construcción social y percepción del riesgo, incluidos los significados y valores de los actores. Para los propósitos de este trabajo, esta perspectiva es importante porque reconoce el papel de aspectos como género, clase social y generación, en el contexto de las relaciones de poder, los aspectos psicosociales de las relaciones y los marcos socioculturales.

Dado que son múltiples los factores que influyen en la forma como tomamos posición frente 
al riesgo, la interacción en los grupos humanos, en particular la manera en que las diferentes generaciones se transmiten sus saberes, constituyen un aspecto de especial interés. La percepción no está aislada de las experiencias socializadoras de los individuos en sus respectivos contextos socioculturales, como se observa a continuación.

\section{ALGUNOS ESTUDIOS SOBRE RELACIONES INTERGENERACIONALES EN EL ÁMBITO DE LA PERCEPCIÓN DEL RIESGO DE DESASTRE}

Los estudios sobre riesgos de desastres y percepción de riesgo que involucran la perspectiva intergeneracional son escasos (e. g., Mardones, Rueda y Guzmán, 2011; Mardones, 2013; Tanner, Rodríguez y Lazcano, 2008). En el campo de las percepciones, se encuentra el estudio realizado por Valckx (2004), dirigido por López Vázquez, con habitantes de Atlixco, Puebla, localidad que se encuentra entre las zonas de riesgo del volcán Popocatépetl en México. Se midieron las diferencias en cuanto a la percepción del riesgo en tres grupos etarios: niños, adolescentes y adultos. Las variables evaluadas fueron las atribuciones antropomórficas relacionadas con las manifestaciones de peligro — personalidad del volcán y acciones humanizadas del volcán-, la sobreestimación y la subestimación del peligro volcánico. En los resultados de este estudio, se muestra que los niños hacen más atribuciones antropomórficas que los adolescentes y adultos, los adolescentes tienden más a sobreestimar el peligro que los niños y los adultos. En los grupos etarios no hubo diferencias significativas en la subestimación. El estudio demuestra la existencia de diferencias en las atribuciones al volcán y en las interpretaciones sobre el peligro. Si la percepción del riesgo es diferente en cada grupo etario, no puede considerarse el mismo tipo de estrategia de comunicación del riesgo para toda la población (Valckx, 2004). López Vázquez
(2009) también recomendó realizar estudios que incluyeran niños, pues pocos trabajos sobre el tema lo hacen.

En dos estudios realizados en la zona de amenaza volcánica alta del Volcán Galeras, en Colombia, se coincidió en que los niños, adolescentes y jóvenes reciben constantemente informaciones diversas y con frecuencia contradictorias por parte de padres, familiares, amigos, docentes, representantes de instituciones para la gestión del riesgo, adultos mayores de las comunidades y medios de comunicación, entre otras fuentes. En cuanto a los adultos mayores, presentan un alto arraigo territorial, fruto de su larga convivencia con el territorio y de la fuerza de su herencia ancestral. En esta zona fue evidente que en cada familia existen posicionamientos distintos alrededor de la posibilidad de un reasentamiento. Para los más jóvenes es más fácil concebir un posible reasentamiento en contraste con los mayores, quienes por lo general lo consideran inviable (Ojeda, 2008; 2011).

En un estudio realizado por Guzmán (2012), se trató tanto la construcción social de la vulnerabilidad como la construcción social de la percepción del riesgo en dos comunidades de Yucatán, México, azotadas por el huracán Isidoro. Aquí la percepción se entiende como los acuerdos sociales o explicaciones elaborados en una comunidad en torno al riesgo, mediados por la relación con la naturaleza y la interacción social que generan construcciones culturales. El conocimiento se socializa de una generación a otra y es el punto de partida para producir las relaciones con el entorno.

Estos estudios incluyen aspectos generacionales en la percepción del riesgo de desastre, identifican características de distintos grupos etáreos, transmisión de patrones entre generaciones y posicionamientos diversos frente al riesgo. Sólo la investigación de Valckx (2004) tuvo como propósito enfocar características psicosociales de los grupos etarios seleccionados, pero no estudió la 
influencia entre generaciones. En conclusión, si bien estos antecedentes son importantes para mostrar la importancia del tema, es necesario desarrollar estudios que pongan énfasis en las relaciones intergeneracionales como categorías o variables involucradas en la construcción social de la percepción del riesgo de desastre.

\section{Conclusiones y recomendaciones}

Las relaciones intergeneracionales constituyen un aspecto fundamental en el surgimiento y desarrollo de la percepción de los riesgos de desastres; sin embargo, es un tema poco explorado. Por ello, en este trabajo se toman algunos aportes de disciplinas de las ciencias sociales, como la psicología social, la sociología y la antropología, que pueden ser un marco para su desarrollo. El tema contiene un potencial importante para profundizar con una perspectiva interdisciplinaria, en aras de lograr un mejor entendimiento del comportamiento humano frente a los riesgos y desastres, con la finalidad de generar acciones eficaces para su prevención.

La existencia de las relaciones intergeneracionales implica que las comunidades poseen una memoria colectiva, pues cada generación retoma las enseñanzas de sus antecesores y deja un legado a sus sucesores, lo que constituye marcos de referencia para la construcción social de la realidad, por ende del riesgo y su percepción. Las generaciones construyen la percepción del riesgo a partir de la tradición oral como parte de la sabiduría popular, que es importante preservar y revitalizar a la luz de los nuevos tiempos. Las estrategias de prevención del riesgo deben ser cercanas a la población e integrar sus códigos psicosocioculturales para que se reconozca y se identifique.

Es posible aprovechar estos legados a favor de la gestión de los riesgos. Primero, es necesario conocer mejor los aspectos implicados en su construcción.
En seguida, articularlos con las estrategias educativas participativas para la prevención de los riesgos y de desastres, en búsqueda de una conjugación de saberes populares y técnico-científicos.

En este orden de ideas, los diálogos de saberes son además una vía para el acercamiento entre distintas generaciones y para promover encuentros entre grupos etarios y roles sociales - por ejemplo, jóvenes y adultos, padres e hijos, maestros y alumnos, funcionarios de gobierno y población civil-, de manera que las percepciones sobre el riesgo se dinamicen y se enriquezcan, a la par que movilicen los marcos institucionales de la familia, la escuela y la comunidad, para llegar a propuestas de gestión del riesgo incluyentes y participativas. Esto también contribuye al empoderamiento de la sociedad ante la necesidad de afrontar de la mejor manera posible los riesgos de desastres a los que está expuesta.

La ciencia y el saber popular coexisten y a la vez entran en conflicto ante temas complejos, como los riesgos de desastres. El diálogo entre las generaciones puede, por un lado, conservar la memoria histórica de acontecimientos pasados que los más jóvenes no vivieron o no recuerdan, y por el otro, enriquecer el conocimiento de los mayores con las nuevas tecnologías y conocimientos sobre el riesgo al que estén expuestos.

Las generaciones cambian a la par que los acontecimientos históricos y contextuales de los riesgos de desastres, por lo tanto, los significados también. A partir de los trabajos intergeneracionales es posible conocer los aspectos de la percepción del riesgo que se mantienen y los que se transforman, así como las circunstancias en las que se producen estos movimientos. Estudiar las generaciones implica identificar y caracterizar distintos momentos históricos de los peligros a los cuales nos enfrentamos y aportar nuevos elementos para profundizar en las características de las etapas del desastre: antes, durante y después. A su vez, enmarcar históricamente estas etapas desde una perspectiva intergeneracional 
facilita la comprensión de las implicaciones políticas de la aceptación de un riesgo en cuanto a aspectos como los conflictos generacionales, los movimientos sociales gestados en cada generación, los posicionamientos en las nuevas generaciones y el sistema de valores, los cuales pueden favorecen o bloquear los esfuerzos de prevención, pues si un hábito no ha pasado al sistema de valores, no será reproducido ni transmitido entre las generaciones.

En el diálogo de saberes se pueden reconocer las interacciones entre sectores sociales - medios de comunicación, gobierno, organizaciones educativas y de la sociedad civil, grupos comunitariosy proponer estrategias de comunicación de riesgos pertinentes y más efectivas. Se busca una ampliación de los marcos de referencia para interpretar los peligros en un proceso educativo incluyente de las dimensiones presentes en el ser humano y la sociedad: simbólica, espiritual, material, científica, política, entre otras.
Por último, es importante reconocer la importancia de las relaciones intergeneracionales en las agendas políticas sobre la gestión integral de riesgos de desastres. Pueden retomarse algunos elementos del Marco de Sendai para la Reducción del Riesgo de Desastres 2015-2030 (ONU, 2015), que considera entre sus prioridades el entendimiento de éstos. Algunos elementos de este marco permiten sustentar esta idea, entre ellos: a) la necesidad de fortalecer las comunidades desde sus experiencias y conocimientos ancestrales; b) la importancia de considerar las causas de fondo y los procesos implicados en la exposición a riesgos, entre ellos, las percepciones, capacidades y potencialidades locales, las relaciones de género y generación; c) el principio del compromiso y alianza de toda la sociedad, a partir de la participación, inclusión, accesibilidad y no discriminación; incluyendo una perspectiva etaria, entre otras. Estos recientes lineamientos constituyen un contexto posibilitador para la gestión de riesgos de desastres. D

\section{Bibliografía}

Beck, Ulrich, 1998, La sociedad del riesgo. Hacia una nueva modernidad, Paidós, Barcelona.

—_, 2002, La sociedad del riesgo global, Siglo XXI Editores, Madrid.

, 2008, La sociedad del riesgo mundial. En busca de la seguridad perdida, Paidós, Barcelona.

, 2010, Risk Society: Towards a New Modernity, Sage, Londres.

Berger, Peter y Thomas Luckmann, 1968, La construcción social de la realidad, Amorrortu, Buenos Aires.

Botero, Patricia, Mónica Vega y Mauricio Orozco, 2012, "Relaciones intergeneracionales: implicaciones en procesos de formación política en jóvenes", en Revista Latinoamericana de Ciencias Sociales, Niñez y Juventud, vol. 10, núm. 2, pp. 897-911.

Braz, Ana, Camila Cômodo, Zilda del Prette, Almir del Prette y Anne Fontaine, 2013, "Habilidades sociales e intergeneracionalidad en las relaciones familiares", en Apuntes de Psicología, vol. 31, núm. 1, pp. 77-84.

Briones, Fernando, 2005, "La complejidad del riesgo: un análisis transversal”, en Revista de la Universidad Cristóbal Colón, año III, núm. 20, pp. 9-17.

Caballero, Manuela y Artemio Baigorri, 2013, “¿Es operativo el concepto de generación?”, en Aposta, núm. 56, pp. 1-45.

Caballero, Martha, 2014, Tres tiempos: cambio social en tres generaciones de mujeres en México, AM Editores/Universidad Autónoma del Estado de Morelos, Cuernavaca.

Cardona, Omar (dir.), 2003, La noción de riesgo desde la perspectiva de los desastres. Marco conceptual para su gestión integral, Banco Interamericano de Desarrollo/Universidad Nacional de Colombia-Manizales, Manizales.

—_ 2007, Indicadores de riesgo de desastre y de gestión de riesgos. Programa para América Latina y el Caribe. Informe resumido, Banco Interamericano de Desarrollo, Washington, D. C. 
Cardona, Omar, Juan Bertoni, Tony Gibss, Michel Hermelin y Allan Lavell, 2010, Entendimiento y gestión del riesgo asociado a las amenazas naturales: un enfoque científico integral para América Latina y el Caribe, International Council for Science-Regional Office for Latin America and the Caribbean/Consejo Nacional de Ciencia y Tecnología, Río de Janeiro y México.

Castillo, Óscar, 2014, "La construcción social y los imaginarios sociopolíticos del desastre: una reflexión sobre las inundaciones en Zona Diamante", en El Cotidiano, núm. 187, pp. 249-263.

Castro, Hortensia y Perla Zusman, 2009, "Naturaleza y cultura: ¿dualismo o hibridación? Una exploración por los estudios sobre riesgo y paisaje desde la geografía”, Investigaciones Geográficas, núm. 70, pp. 135-153.

Centro de Coordinación para la Prevención de los Desastres Naturales en América Central (Cepredenac), 2010, Política centroamericana de gestión integrada de riesgo de desastres, Centro de Coordinación para la Prevención de los Desastres Naturales en América Central, Guatemala.

Consejo Andino de Ministros de Relaciones Exteriores, 2009, Estrategia andina para la prevención y atención de desastres, Secretaría General de la Comunidad Andina, Lima.

Cruz, Rocío y Saray Acosta, 2009, "Vocabulario intergeneracional. Un intercambio de mayores y alumnado en las aulas", en Cuestiones Pedagógicas, núm. 20, pp. 247-268.

Donati, Pier Paolo, 1999, “Familias y generaciones”, en Desacatos. Revista de Antropología Social, núm. 2, pp. 27-49.

Douglas, Mary, 1996, La aceptabilidad del riesgo según las ciencias sociales, Paidós, Barcelona.

Douglas, Mary y Aaron Wildavsky, 1982, Risk and Culture. An Essay on the Selection of Technological and Environmental Dangers, University of California Press, Berkeley.

Falcke, Denise y Adriana Wagner, 2003, "La dinámica familiar y el fenómeno de la transgeneracionalidad: definición de conceptos”, en Adriana Wagner (coord.), La transmisión de modelos familiares, CCS, Madrid, pp. 21-43.

Feixa, Carles, 2003, “Del reloj de arena al reloj digital”, en Jóvenes. Revista de Estudios sobre Juventud, vol. 7, núm. 19, pp. 6-27.

Ferrari, M. Paula, 2010, “Percepción social del riesgo: problemáticas costeras y vulnerabilidades en playa Magagna (Chubut)", en Huellas, núm. 15, pp. 13-33.

Fischhoff, Baruch y John Kadvany, 2013, Riesgo: una breve introducción, Alianza, Madrid.

Fischhoff, Baruch, Paul Slovic, Sarah Lichtenstein, Sthepen Read y Barbara Combs, 2000, "How Safe Is Safe Enough? A Psychometric Study of Attitudes Toward Technological Risks and Benefits”, en Paul Slovic (coord.), The Perception of Risk, Earthscan, Londres, pp. 80-103.

Gallego, Gisella, 2011, Acercamiento cultural intergeneracional. Propuestas desde la comunicación para la interrelación entre jóvenes y adultos mayores en Segovia, trabajo final de máster en comunicación con fines sociales: estrategias y campañas, Universidad de Valladollid, Valladolid.

García Acosta, Virginia, 2005, “El riesgo como construcción social y la construcción social de riesgos”, en Desacatos. Revista de Antropología Social, núm. 19, pp. 11-24.

Gellert de Pinto, Gisela, 2012, "El cambio de paradigma: de la atención de desastres a la gestión del riesgo", en Boletín Científico Sapiens Research, vol. 2, núm. 1, pp. 13-17.

González, Jerónimo y Raquel de la Fuente, 2008, "Relevancia psico-socio-educativa de las relaciones generacionales abuelo-nieto", en Revista Española de Pedagogía, núm. 239, pp. 103-118.

Gutiérrez, María y Gemma Herráiz, 2009, "La sinergia intergeneracional”, en Espai Social, núm. 9, pp. 25-31. Disponible en línea: <http://www. espaisocial.net/docs/revistas/ espai_social_09.pdf>.

Guzmán, Gertrudis, 2012, La construcción social del riesgo del desastre en el sureste de México: el huracán Isidoro en dos comunidades de Yucatán, tesis de maestría en administración integral del ambiente, El Colegio de la Frontera Norte/Centro de Investigación Científica y de Educación Superior de Ensenada, Tijuana.

Hacking, lan, 1999, The Social Construction of What?, Harvard University Press, Cambridge.

_ 2001, ¿La construcción social de qué?, Paidós, Barcelona.

Harwood, Jake, 2002, "Comunicación intergeneracional entre extraños y entre miembros de la familia”, en Revista Latinoamericana de Psicología, año 34, núms. 1-2, pp. 75-82.

Intergovernmental Panel on Climate Change (IPCC), 2014, Climate Change 2014: Synthesis Report. Contribution of Working Groups I, II and III to the Fifth Assessment Report of the Intergovernmental Panel on Climate Change [Rajendra K. Pachauri y Leo A. Meyer (eds.)], Intergovernmental Panel on Climate Change, Ginebra.

Laín, Pedro, 1945, Las generaciones en la historia, Instituto de Estudios Políticos, Madrid. 
Lavell, Thomas Allan, 1993, "Ciencias sociales y desastres naturales en América Latina: un encuentro inconcluso", en Eure, Revista Latinoamericana de Estudios urbano Regionales, vol. 19, núm. 58, pp. 73-84.

—_ 2005, Los conceptos, estudios y práctica en torno al tema de los riesgos y desastres en América Latina: evolución y cambio, 19802004, Facultad Latinoamericana de Ciencias Sociales. Disponible en línea: <http://bibliotecavirtual.clacsoorg.ar/ar/libros/flacso/ secgen/lavell.pdf>.

Leccardi, Carmen y Carles Feixa, 2011, “El concepto de generación en las teorías sobre la juventud”, en Última Década, núm. 34 , pp. 11-32.

López, Alejandra, 2011, Trama y urdimbre del riesgo a desastre en una comunidad campesina: Santiago Xalitzintla, Puebla, tesis de maestría en desarrollo rural, Universidad Autónoma Metropolitana, México.

López-Moreno, Ligia y Sara Alvarado, 2011, "Emergencia de las relaciones intergeneracionales en una escuela pública urbana”, en Revista Latinoamericana de Ciencias Sociales, Niñez y Juventud, vol. 9, núm. 1, pp. 255-268.

López Vázquez, Esperanza, 2009, "Risk Perception and Coping Strategies for Risk from Popocatépetl Volcano, Mexico", en Geofísica Internacional, vol. 48, núm. 1, pp. 301-315.

Lüscher Kurt, Andreas Hoff, Giovanni Lamura, Marta Renzi, Mariano Sánchez, Gil Viry, Éric Widmer, Andrzej Klimczuk y Paulo de Salles Oliveira, 2015, Generaciones, relaciones intergeneracionales, politica generacional. Un compendio multilingüe, Generationes, International Network for the Study of Intergenerational Issues/Universität Konstanz. Disponible en línea: <www.generationen-compendium.de>

Mardones, Rodrigo, 2013, "Radio, jóvenes y participación: una experiencia de investigación acción participativa (IAP) en el contexto de posterupción volcánica en Chaitén”, en Revista Latinoamericana de Psicología Social lgnacio Martín-Baró, vol. 2, núm. 2, pp. 197-213.

Mardones, Rodrigo, Sebastián Rueda y Marcela Guzmán, 2011, “Tejiendo vínculos: una mirada a la organización 'Renacer de Chaitén’ de la tercera edad en un contexto de posdesastre", en Cuadernos de Crisis y Emergencias, vol. 2, núm. 10, pp. 1-22.

Martin, Marco, 2008, "La teoría de las generaciones de Ortega y Gasset: una lectura del siglo xxl”, en Tiempo y Espacio, año 17, vol. 20, pp. 98-110.

Maskrey, Andrew, 1997, “Comunidad y desastres en América Latina: estrategias de intervención”, en Allan Lavell (comp.), Viviendo en riesgo: comunidades vulnerables y prevención de desastres en América Latina, Red de Estudios Sociales en Prevención de Desastres en América Latina/Facultad Latinoamericana de Ciencias Sociales/Centro de Prevención de Desastres Naturales en Centroamérica, Lima, pp. 5-32.

Mead, Margaret, 1970, Cultura y compromiso. El mensaje de la nueva generación, Granica, Buenos Aires.

__, 1977, Cultura y compromiso. El mensaje de la nueva generación, Granica, Barcelona.

Méndez, Ana María e Irma Castro, 2011, "Aprendizajes intergeneracionales en los surcos de chile y jitomate. Experiencias con niñas, niños y mujeres de familias jornaleras agrícolas migrantes", en Decisio, septiembre-diciembre, pp. 78-85.

Moreno, Almudena, 2011, "La reproducción intergeneracional de las desigualdades educativas: límites y oportunidades de la democracia", en Revista de Educación, núm. extraordinario, pp. 183-206.

Nadorovsky, Mariano, 2014, “Infancia, pasado y nostalgia. Cambios en la tradición intergeneracional”, en Revista Brasileira de Educação, vol. 14, núm. 2, pp. 191-214.

Nina, Esteban, Santiago Grillo y Carlos Alonso, 2003, "Movilidad social y transmisión de la pobreza en Bogotá”, en Economía y Desarrollo, vol. 2, núm. 2, pp. 119-156.

Nussbaum, Silvia, 2009, "Identificaciones alienantes y repetición. Una contribución acerca de la transmisión intergeneracional”, en Psicoanálisis, vol. XXXI, núm. 1, pp. 153-166.

Ojeda, Elizabeth, 2008, "Problemática psicosocial en comunidades residentes en la zona de amenaza volcánica alta del Volcán Galeras: una perspectiva para el acompañamiento psicosocial desde la psicología comunitaria", en Universidad y Salud, año 8, vol. 1, núm. 10, pp. 45-64.

__. 2011, Representaciones sociales de comunidad en la Parcialidad Indigena de Jenoy, tesis de magíster en etnoliteratura, Universidad de Nariño, Pasto.

Organización de las Naciones Unidas (ONu), 2015, Marco de Sendai para la Reducción del Riesgo de Desastres 2015-2030, Organización de las Naciones Unidas, Ginebra. Disponible en línea: <http://www.unisdr.org/files/43291_spanishsendaiframeworkfordisasterri.pdf>.

Ortelli, Paola, 2012, "Aprender de los mayores: un intercambio intergeneracional en los Altos de Chiapas", en Chasqui. Revista Latinoamericana de Comunicación, núm. 120, pp. 41-45.

Pérez-Sánchez, Rolando y Vanessa Smith-Castro, 2008, “Comunicación intergeneracional: el Cuestionario de Percepción de la Comunicación Intergeneracional (CPCI) en el contexto costarricense”, en Interamerican Journal of Psychology, vol. 42, núm. 3, pp. 589-603. 
Ramírez, Clemencia, 2003, "La transmisión intergeneracional, la clase del vínculo y los factores intrapersonales como predictores de la coocurrencia de comportamientos violentos y adictivos en jóvenes", en Acta Colombiana de Psicología, núm. 9, pp. 51-69.

Sabogal, Alba, Beatriz Rojas, Carolina Díaz, Martha Ochoa, Nilson Correa y Samuel Ospina, 2006, "Evaluación dimensional del riesgo percibido en el barrio La Dulcera, Municipio de Pereira”, en Scientia et Technica, vol. XII, núm. 30, pp. 421-426.

Sáez, Juan, 2009, "La intergeneracionalidad o la potencialidad de un concepto inexplorado”, en Espai Social, núm. 9, pp. 4-7. Disponible en línea: <http://www.espaisocial.net/docs/revistas/espai_social_09.pdf>.

Sánchez, Mariano, 2009, "La necesidad de los programas intergeneracionales. Ayer, hoy, mañana”, en Espai Social, núm. 9, pp. 8-12. Disponible en línea: <http://www.espaisocial.net/docs/revistas/espai_social_09.pdf>.

Sarasty-Almeida, Saira, María González-Gómez e Iván Velasco-Charfuelán, 2014, "Red de apoyo social desde el encuentro intergeneracional”, en Universidad y Salud, vol. 16, núm. 1, pp. 22-34.

Schutz, Alfred, 1974, El problema de la realidad social, Amorrortu, Buenos Aires.

Siegrist, Michael y George Cvetkovich, 2000, "Perception of Hazards: The Role of Social Trust and Knowledge", en Risk Analysis, vol. 20, núm. 5, pp. 713-719

Sjöberg, Lennart, 2000, "Factors in Risk Perception", en Risk Analisys, vol. 20, núm. 1, pp. 1-11.

Slovic, Paul, 2000, "Perception of Risk”, en Paul Slovic (coord.), The Perception of Risk, Earthscan, Londres, pp. 220-231.

Slovic, Paul y Elki Weber, 2002, "Perception of Risk Posed by Extreme Events", ponencia presentada en la conferencia Risk Management Strategies in an Uncertain World, Palisades, Nueva York, 13 de abril.

Tanner Thomas, Gonzalo Rodríguez y Jimena Lazcano, 2008, "Los niños y niñas, y la gestión de riesgos: un rol clave en la prevención de desastres", en Medio Ambiente y Urbanización, vol. 69, núm. 1, pp. 1-17.

Testaverde, Tommaso, 2012, El concepto de generación en la actividad crítica y teórica de Oreste Macrì, tesis de doctorado en teoría de la literatura y del arte y literatura comparada, Universidad de Granada, Granada.

Tierney, Kathleen, 2014, The Social Roots of Risk. Producing Disasters, Promoting Resilience, Stanford University Press, Stanford.

Urteaga, Eguzki y Andoni Eizagirre, 2013, "La construcción social del riesgo", en Empiria. Revista de Metodología de las Ciencias Sociales, núm. 25, pp. 147-170.

Valckx, Astrid, 2004, Percepción de riesgo volcánico e interpretación de la actividad del Popocatépetl en niños, adolescentes y adultos, tesis de licenciatura en psicología, Universidad de las Américas, Puebla.

Vargas, Luz María, 1994, “Sobre el concepto de percepción”, en Alteridades, vol. 4 núm. 8, pp. 47-53.

Vera, Gabriela, 2009, "Ancianos, tiemperos y otras figuras de autoridad en dos comunidades del volcán Popocatépetl. La otra visión del riesgo volcánico", en Jesús Manuel Macías (coord.), La disputa por el riesgo en el volcán Popocatépetl, Centro de Investigaciones y Estudios Superiores en Antropología Social, México, pp. 99-116.

Wachinger, Gisella y Ortwin Renn, 2010, Risk Perception and Natural Hazards, CapHaz-Net WP3 Report/Dialogik Non-Profit Institute for Communication and Cooperative Research, Stuttgart. Disponible en línea: <https://www.researchgate.net/profile/Tracey_Coates/ publication/228827276_Risk_perception_of_natural_hazards/links/00b49519f2905da3f5000000.pdf>.

Wachinger, Gisella, Ortwin Renn, Chloe Begg y Christian Kuhlicke, 2013, "The Risk Perception Paradox-Implications for Governance and Communication of Natural Hazards”, en Risk Analysis, vol. 33, núm. 6, pp. 1049-1065.

Wagner, Adriana, Juliana Carmona y Denise Falcke, 2003, “Transgeneracionalidad y educación: ¿cómo se perpetúa la familia?”, en Adriana Wagner (coord.), La transmisión de modelos familiares, CCS, Madrid, pp. 79-93.

Werba, Alicia, 2002, "Transmisión entre generaciones. Los secretos y los duelos ancestrales”, en Psicoanálisis, vol. XXIV, núms. 1-2, pp. 295 -313. 